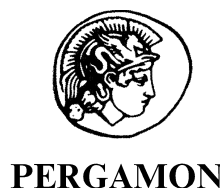

PERGAMON
Journal of the Mechanics and Physics of Solids 48 (2000) 1107-1131
JOURNAL OF THE

MECHANICS AND

PHYSICS OF SOLIDS

www.elsevier.com/locate/jmps

\title{
Crack patterns in thin films
}

\author{
Z. Cedric Xia ${ }^{\text {a }}$, John W. Hutchinson ${ }^{\mathrm{b}, *}$ \\ ${ }^{a}$ Ford Research Laboratory, Manufacturing Systems Department, Dearborn, MI 48121, USA \\ ${ }^{\mathrm{b}}$ Harvard University, Division of Engineering and Applied Sciences, 29 Oxford Street, Cambridge, \\ MA 02138, USA
}

Received 16 February 1999

\begin{abstract}
A two-dimensional model of a film bonded to an elastic substrate is proposed for simulating crack propagation paths in thin elastic films. Specific examples are presented for films subject to equi-biaxial residual tensile stress. Single and multiple crack geometries are considered with a view to elucidating some of the crack patterns which are observed to develop. Tendencies for propagating cracks to remain straight or curve are explored as a consequence of crack interaction. The existence of spiral paths is demonstrated. (C) 2000 Elsevier Science Ltd. All rights reserved.
\end{abstract}

Keywords: Crack propagation; Thin films; Integral equations

\section{Introduction}

Films and coatings bonded to substrates often develop in-plane tensile stresses large enough to cause cracking. A film deposited at a high temperature and then cooled will develop biaxial in-plane tensile stresses if the thermal expansion coefficient of the film exceeds that of the substrate. This is usually the case for metal or polymer films deposited on ceramic substrates, and it is often the situation for glazes on pottery. Tensile stresses develop in coatings such as paints and lacquers due to solvent evaporation producing a tendency for the coating to

\footnotetext{
* Corresponding author. Tel.: +1-617-495-2848; fax: +1-617-495-9837.

E-mail address: hutchinson@husm.harvard.edu (J.W. Hutchinson).
} 
shrink were it is not bonded to the substrate. In much the same way, tensile stresses develop in a constrained layer of mud as drying takes place.

Glaze cracks, mud cracks, crack formations such as the Devils Postpile and the Giant's Causeway, and other crack patterns have long held a fascination for mankind. Nevertheless, in most instances, the understanding underlying the evolution of such crack patterns is qualitative at best. In this paper, the focus will be on cracking behavior in biaxially stressed thin elastic films and coatings that are well bonded to thick elastic substrates. A two-dimensional model for the filmsubstrate system is proposed which permits an analytical investigation of a wide variety of film cracking phenomena. A number of solutions based on the model will be presented in this paper with the intent of providing the mechanics underlying crack path and pattern evolution. These include several interaction effects, such as conditions establishing crack spacing and the behavior of one crack advancing toward another. Insight is also provided into the tendency for cracks to kink or curve due to the presence of a neighboring crack. One intriguing theoretical prediction is the existence of spiral crack paths under conditions where the film is subject to equi-biaxial tension. Spiral paths in films do not appear to be commonly observed, but a good example is contained in an early paper by Argon (1959). Dillard et al. (1994) have reported many examples of spiral cracks in thin brittle adhesive layers bonding together glass plates. Observations of other unusual crack paths and patterns in thin films and coatings can be found in articles by Chen and Chen (1995) and Garino (1990).

Crack propagation in a film bonded to a substrate is a three-dimensional process. As depicted in Fig. 1, a crack initiates at a flaw and spreads by channeling. One of the few fully three-dimensional studies of film cracking is that of Nakamura and Kamath (1992) who analyze an isolated finite length throughfilm crack, including its approach to steady-state propagation wherein conditions at the crack edge become independent of the length of the crack. Remarkably, their results show that a crack whose length is only slightly greater than several film thicknesses is already close to steady state, for the case of a film on a rigid substrate. The energy release rate $G$ of a steady-state channeling crack in Fig. 1 can be obtained from a two-dimensional plane strain analysis, even though the process itself is three-dimensional. By considering the energy difference between sections of the film/substrate system far ahead and far behind the crack edge, one can rigorously obtain results for the energy release rate averaged over the crack edge in terms of plane strain solutions for cracked films. Solutions for steady-state channeling in films have been presented by Beuth (1992), and further relevant mechanics and results are summarized in the review article by Hutchinson and Suo (1992).

Beuth's (1992) result for the energy release rate averaged over the advancing front of a semi-infinite isolated crack is

$$
G=\frac{\pi}{2} \frac{\left(1-v^{2}\right) h \sigma^{0^{2}}}{E} g(\alpha, \beta)
$$


where $h$ is the film thickness, $E$ and $v$ are the Young's modulus and Poisson's ratio of the film, respectively. The Dundur's parameters, $\alpha$ and $\beta$, characterizing the elastic mismatch between the film and the substrate are

$$
\alpha=\frac{\bar{E}-\bar{E}_{\mathrm{s}}}{\bar{E}+\bar{E}_{\mathrm{s}}} \quad \text { and } \quad \beta=\frac{1}{2} \frac{\mu\left(1-2 v_{\mathrm{s}}\right)-\mu_{\mathrm{s}}(1-2 v)}{\mu\left(1-v_{\mathrm{s}}\right)+\mu_{\mathrm{s}}(1-v)}
$$

where $E_{\mathrm{s}}$ and $v_{\mathrm{s}}$ are the elastic constants of the substrate, respectively, $\mu=$ $E /(2(1+v))$ denotes a shear modulus, and $\bar{E}=E /\left(1-v^{2}\right)$ is a plane strain tensile modulus. Eq. (1) applies for cracks extending down to the film/substrate interface with $\sigma^{0}$ as the uniform prestress in the film acting normal to the crack line. The prestress has no shear component acting parallel to the crack, and thus mode-I conditions hold on the crack edge. The function $g(\alpha, \beta)$ is presented in Fig. 1.

With $\Gamma_{\mathrm{c}}$ as the mode-I fracture toughness of the film measured in units of energy per unit area, the condition for propagation of an isolated crack across a brittle film is

$$
G=\Gamma_{\mathrm{c}}
$$

This condition provides a robust condition to design against extensive film cracking because short cracks and crack-like flaws will have energy release rates which fall below the steady-state rate (1). When only small flaws are present, film cracks will not begin to spread until the prestress and/or film thickness exceeds the

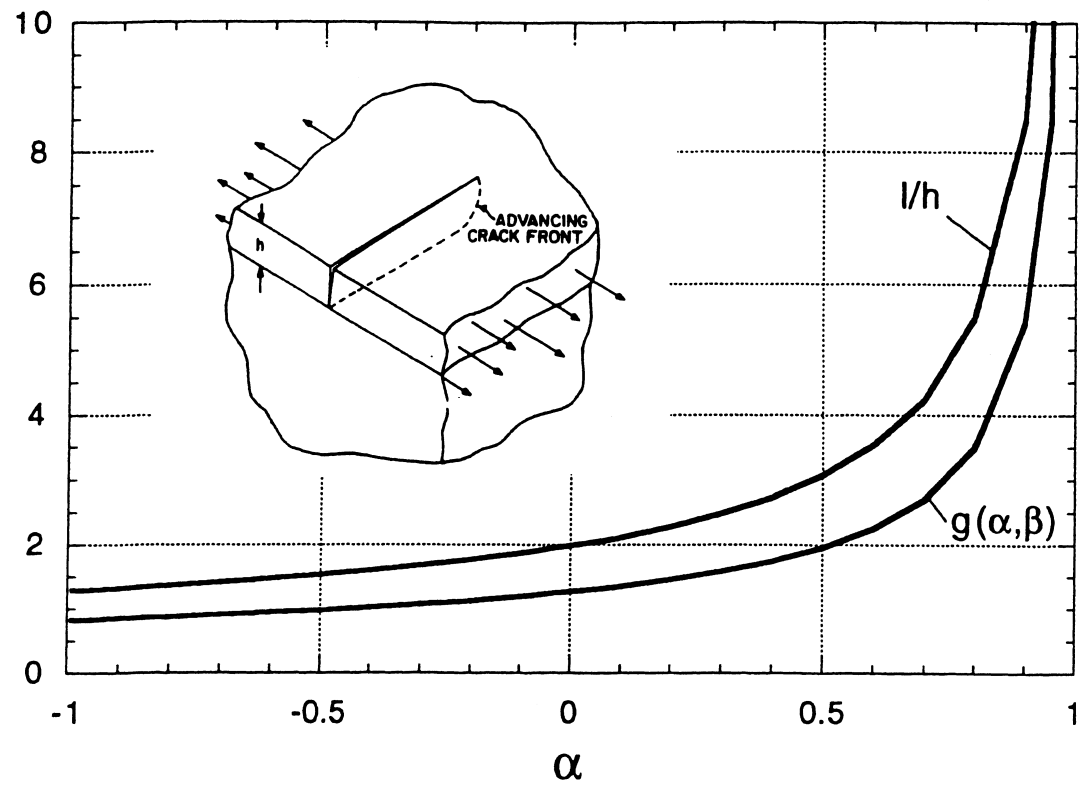

Fig. 1. Steady-state channeling crack in a thin film. The function $g(\alpha, \beta)$ and the normalized length defining the in-plane resistance of the substrate, $l / h$, for $\beta=0$ (the dependence on $\beta$ is weak). 
steady-state requirement (3). On the other hand, the Nakamura-Kamath study shows that any crack-like flaws must be small compared to the film thickness if crack spreading is likely to be postponed to stress/thickness levels significantly above the steady-state requirement (3). Film cracking is frequently influenced by environmental factors, producing some degree of time-dependence of crack growth. Humidity affects the propagation of cracks in glasses, and curing and drying are inherently time-dependent.

The steady-state energy release rate (1) is modified by various effects. If the substrate is very stiff compared to the film, the channeling crack may not reach the interface with the substrate. This possibility and the determination of the depth attained by the crack is discussed by Beuth (1992). Conversely, depending on the elastic mismatch and the toughness of the substrate relative to the film, the crack may penetrate into the substrate (Ye et al., 1992). Another commonly observed phenomenon is film debonding accompanying the channeling crack (Ye et al., 1992) which can occur if the interface toughness is sufficiently low compared to that of the film and substrate. There exists a range of the interface toughness, relative to the film toughness, such that the debonded region left behind by the advancing crack front has a well defined width on either side of the film crack. Plastic yielding in the substrate induced by the film crack also results in a modification of $G$ (Hu and Evans, 1989; Beuth and Klingbeil, 1996).

Each of the above mentioned effects can influence film crack interaction and the paths that cracks follow. There are instances, for example, in which a propagating film crack induces a interface debond on one side of the crack but not the other. This produces a strong asymmetry with respect to the crack tip, causing the crack to follow a curved trajectory. Such auxiliary effects will not be considered in this paper. Attention will be limited to film cracks unaccompanied by substrate cracking, interface debonding or substrate yielding.

\section{The model}

\subsection{Formulation}

A uniform, isotropic elastic film bonded to an isotropic substrate is modeled as a sheet of thickness $h$ attached to an elastic foundation. Prior to cracking, the film is uniformly stressed such that $\sigma_{\alpha \beta}=\sigma_{\alpha \beta}^{0}$. A plane stress approximation is used to describe the in-plane deformation of the film in the presence of cracks, with $u_{\alpha}\left(x_{1}, x_{2}\right)$ as the in-plane displacements averaged through the thickness of the film and measured relative to the uniform prestressed state. The associated average inplane strains are. $\varepsilon_{\alpha \beta}=1 / 2\left(u_{\alpha, \alpha}+u_{\beta, \beta}\right)$. The average stresses in the film are

$$
\sigma_{\alpha \beta}=\sigma_{\alpha \beta}^{0}+\Delta \sigma_{\alpha \beta}
$$

where $\Delta \sigma_{\alpha \beta}$ is the average through the thickness of the stress changes due to cracking. The average stress changes are related to the average strain changes by 


$$
\Delta \sigma_{\alpha \beta}=\frac{E}{1-v^{2}}\left[(1-v) \varepsilon_{\alpha \beta}+v \varepsilon_{\gamma \gamma} \delta_{\alpha \beta}\right]
$$

where any effect of $\Delta \sigma_{33}$ has been neglected. Greek subscripts range from 1 to 2 in the usual convention for plane stress.

Denote the in-plane components of the restoring force per unit area exerted on the film by the substrate by $f_{\alpha}$. Equilibrium requires that the stress averages exactly satisfy $h \sigma_{\alpha \beta, \beta}+f_{\alpha}=0$. The elastic restoring force per unit area exerted by the substrate on the film is modeled by $f_{\alpha}=-k u_{\alpha}$ where the spring constant $k$ will be identified later. The associated Navier equations governing the displacements of the film are

$$
\frac{1}{2}(1-v) u_{\alpha, \beta \beta}+\frac{1}{2}(1+v) u_{\beta, \beta \alpha}=\frac{\left(1-v^{2}\right) k}{E h} u_{\alpha}
$$

A traction-free crack must satisfy $\sigma_{\alpha \beta} n_{\beta}=0$ at every point along the crack, where $n_{\beta}$ is the unit normal to the crack line, such that the average traction changes cancel the pre-tractions, i.e. $\Delta \sigma_{\alpha \beta} n_{\beta}=-\sigma_{\alpha \beta}^{0} n_{\beta}$.

According to the model, an elastic substrate does not alter the character of the dominant singular behavior at the tip of the crack in the film. The stress changes at the crack tip governed by Eq. (5) have the conventional mode I and II inverse square root singularities of plane stress with amplitudes $K_{\mathrm{I}}$ and $K_{\mathrm{II}}$ defined in the standard manner. The strain energy per area per unit thickness of film is

$$
\begin{aligned}
& W=W^{0}+\Delta W \\
& \text { where } \quad \Delta W=\left[\sigma_{\alpha \beta}^{0} \varepsilon_{\alpha \beta}+\frac{1}{2} \sigma_{\alpha \beta} \varepsilon_{\alpha \beta}\right]+\frac{1}{2}\left(\frac{k}{h}\right) u_{\alpha} u_{\alpha}
\end{aligned}
$$

with $W^{0}$ as the elastic energy density stored in the film prior to cracking. The elastic energy per unit area in the model film/substrate system is $h W$. Energy contributions in the film from through-thickness variations departing from the average stresses and strains are neglected in Eq. (6). With the strain energy of the system defined as the area integral of Eq. (6), $\int h W \mathrm{~d} A$, the principle of minimum potential energy for the model leads precisely to Eq. (5) as the associated Euler equations. The energy release rate of the crack (energy release per unit of crack advance per unit thickness of film) is related to the stress intensity factors by the classical plane stress relation $G=\left(K_{\mathrm{I}}^{2}+K_{\mathrm{II}}^{2}\right) / E$. A path-independent $J$-integral exists for the model whose value coincides with $G$ :

$$
J=\int_{C}\left[W n_{1}-\sigma_{\alpha \beta} n_{\beta} u_{\alpha, 1}\right] \mathrm{d} s
$$

where $C$ is any contour circling the tip in the counter-clockwise sense with $n_{\alpha}$ as its outward unit normal and $\mathrm{d} s$ as its element of length. The $x_{1}$-axis must be aligned parallel to the crack line at the tip.

The fracture behavior of the film is assumed to be isotropic with $\Gamma_{\mathrm{c}}$ as the 
mode-I toughness. In applying the model to predict a crack propagation path under quasi-static conditions, the path is required to evolve such that pure mode-I conditions $\left(K_{\mathrm{II}}=0\right)$ are maintained at the tip with $G=\Gamma_{\mathrm{c}}$. A pre-existing film crack subject to increasing prestress may experience combined mode-I and -II conditions at its tip. The crack will initiate growth by kinking in the direction for which $K_{\text {II }}$ of the putative crack increment vanishes. Once growth has been initiated, however, the path is expected to evolve smoothly such that $K_{\mathrm{II}}=0$. The emphasis in this paper in the first instance is not on the prediction of detailed paths, but rather on the production of a variety of crack solutions which will supply qualitative insight into the way crack paths are expected to develop in thin films. To this end, solutions to the model will be presented for a variety of crack geometries in the form of the crack tip intensity measures, $K_{\mathrm{I}}$ and $K_{\mathrm{II}}$. Most of the results will be presented for films subject to an equi-biaxial stress state, $\sigma_{\alpha \beta}^{0}=$ $\sigma^{0} \delta_{\alpha \beta}$.

\subsection{Solution for a semi-infinite straight crack and calibration of model}

Consider an isolated semi-infinite straight crack coincident with the negative $x_{1}$ axis and subject to initial stresses with $\sigma_{12}^{0}=0$. Symmetry dictates that mode-I conditions prevail at the tip. Far behind the tip, the displacement field is independent of $x_{1}$. The solution to the Navier equations (5) is readily obtained as

$$
u_{1}=0 \quad \text { and } \quad u_{2}=\sigma_{22}^{0} \sqrt{\frac{h}{\bar{E} k}} \mathrm{e}^{-\sqrt{\frac{k}{\bar{E} h} x_{2}}}
$$

where $\bar{E}=E /\left(1-v^{2}\right)$. The associated stress changes far behind the tip are

$$
\Delta \sigma_{12}=0, \quad \Delta \sigma_{11}=v \Delta \sigma_{22} \text { and } \Delta \sigma_{22}=-\sigma_{22}^{0} e^{-\sqrt{\frac{k}{\bar{E} h} x_{2}}}
$$

The remote changes do not depend on $\sigma_{11}^{0}$. The energy release rate can be obtained either by a simple energy argument, accounting for the energy change due to a unit advance of the crack tip, or by a direct evaluation of $J$ using a contour remote from the tip. The result is

$$
G=\sqrt{\frac{h}{\bar{E} k}} \sigma_{22}^{0^{2}}
$$

The model is calibrated with the exact solution for the semi-infinite mode-I crack given in Section 1. We choose the substrate spring constant $k$ such that $G$ in Eq. (10) coincides with Eq. (1) with the result that $k$ must satisfy:

$$
\sqrt{\frac{\bar{E}}{k h}}=\frac{\pi}{2} g(\alpha, \beta)
$$


If the film is very compliant relative to the substrate, the film crack may not reach the interface with the substrate, as discussed in Section 1. Beuth's (1992) results may be used to adjust Eq. (11) to account for crack depths which are less than $h$, but this is generally a small correction.

It is convenient to introduce the reference length $l$, characterizing the exponential decay of the changes transverse to the crack in Eqs. (8) and (9):

$$
l \equiv \sqrt{\frac{\bar{E} h}{k}}=\frac{\pi}{2} g(\alpha, \beta) h
$$

In the absence of any elastic mismatch between film and the substrate $(\alpha=\beta=0)$, $l=1.98 h$. From the plot in Fig. 1 , it can be seen that $l$ will greatly exceed the film thickness $h$ when the film is very stiff compared to the substrate $(\alpha \approx 1)$ and will be of the order of $h$ when the film is very compliant relative to the substrate $(\alpha \approx-$ $1)$.

\section{Integral equation formulations}

The Navier equations (5) can be written in a dimensionless form such that $h, E$ and $k$ are absorbed into the dimensionless displacements $u_{\alpha} / l \rightarrow u_{\alpha}$ and coordinates $x_{\alpha} / l \rightarrow x_{\alpha}$ as

$$
\frac{1}{2}(1-v) u_{\alpha, \beta \beta}+\frac{1}{2}(1+v) u_{\beta, \beta \alpha}=u_{\alpha}
$$

In this section and in Appendix A, dimensionless displacements and coordinates will be used, and $k$ will enter into the results only through $l$, which is absorbed into the dimensionless variables. In the other sections, dimensional quantities will be used. With these dimensionless quantities, $\varepsilon_{\alpha \beta}=\frac{1}{2}\left(u_{\alpha, \beta}+u_{\alpha, \beta}\right)$, and the stresses are still given by Eq. (4). Let $\phi$ and $\psi$ be two scalar functions of the coordinates, and introduce the Helmholtz representation, $u_{\alpha}=\phi_{, \alpha}+e_{\alpha \beta} \psi_{, \beta}$, where $e_{\alpha \beta}$ is the permutation tensor. The Navier equations (13) then can be rewritten as

$$
\left(\nabla^{2} \phi-\phi\right)_{, \alpha}+e_{\alpha \beta}\left(\frac{1}{2}(1-v) \nabla^{2} \psi-\psi\right)_{, \beta}=0
$$

with stress changes due to cracking as

$$
\begin{aligned}
& \Delta \sigma_{11}=\left(E /\left(1-v^{2}\right)\right)\left[\phi_{, 11}+v \phi_{, 22}+(1-v) \psi_{, 12}\right] \\
& \Delta \sigma_{22}=\left(E /\left(1-v^{2}\right)\right)\left[\phi_{, 22}+v \phi_{, 11}-(1-v) \psi_{, 12}\right]
\end{aligned}
$$




$$
\Delta \sigma_{12}=(E /(1-v))\left[\phi_{, 12}-\frac{1}{2}\left(\psi_{, 11}-\psi_{, 22}\right)\right]
$$

Integral equation formulations will be used to construct solutions to the problems considered in this paper which cannot be solved analytically. In conventional crack problems in two-dimensional elasticity, integral equations are formulated using dislocations as the kernel functions (e.g. Rice, 1968), leading to integrals with Cauchy singularities. For the present model of film cracking, a formulation based on dislocation doublets is more natural than one based on dislocations. Stresses due to a dislocation in the film are finite at infinity on each side of the dislocation line due to interaction with the underlying substrate, while the stresses produced by a dislocation doublet fall off exponentially far from the doublet. The doublet formulation has integrals with kernels whose singularities are of order $1 / r^{2}$. This class of integral equations has been labeled 'strongly singular', and methods analogous to those for Cauchy equations have recently come available for computing numerical solutions (Kaya and Erdogan, 1987; Willis and NematNasser, 1991).

\subsection{Doublet solution}

To define the dislocation doublets, introduce two dislocations of equal magnitude but opposite sense on the $x_{1}$-axis spaced a distance $2 \varepsilon$ apart. With the amplitude of the dislocation on the right specified by $\mathbf{b}$, bring the dislocations together such that $\mathbf{d}=\lim (2 \varepsilon \mathbf{b})$. (Here, $\mathbf{b}$ and $\mathbf{d}$ are dimensionless. The dimensional quantities are ${ }^{\varepsilon \rightarrow 0}$ scaled by $l$ and $l^{2}$, respectively.) The dominant singularity of the doublet is unaffected by interaction with the substrate and therefore the same as in plane stress. With $(r, \theta)$ as polar coordinates centered at the doublet and $\mathbf{d}=\left(d_{1}, d_{2}\right)$, the dominant singularity is

$$
\begin{aligned}
& \Delta \sigma_{11}=\frac{E}{4 \pi r^{2}}\left[-d_{1}(\sin 2 \theta+\sin 4 \theta)+d_{2} \cos 4 \theta\right] \\
& \Delta \sigma_{22}=\frac{E}{4 \pi r^{2}}\left[-d_{1}(\sin 2 \theta-\sin 4 \theta)+d_{2}(2 \cos 2 \theta-\cos 4 \theta)\right] \\
& \Delta \sigma_{12}=\frac{E}{4 \pi r^{2}}\left[-d_{1} \cos 4 \theta+d_{2}(\sin 4 \theta-\sin 2 \theta)\right]
\end{aligned}
$$

The corresponding Helmholtz functions are

$$
\begin{aligned}
& \phi=\frac{1}{8 \pi}\left[d_{1}(1-v) \sin 2 \theta+d_{2}(2(1+v) \ln r-(1-v) \cos 2 \theta)\right] \\
& \psi=\frac{1}{4 \pi}\left[-d_{1} \cos 2 \theta-d_{2} \sin 2 \theta\right]
\end{aligned}
$$


The full doublet solution satisfies Eq. (14) and must approach Eqs. (16) and (17) as $r \rightarrow 0$. The exact representation for the doublet was found after lengthy manipulation; it is

$$
\begin{aligned}
& \phi=\frac{1}{\pi}\left[-\frac{d_{1}}{\omega^{2}} F(r) \sin 2 \theta+\frac{d_{2}}{4}\left(-(1+v) K_{0}(r)+\frac{4}{\omega^{2}} F(r) \cos 2 \theta\right)\right] \\
& \psi=\frac{1}{\pi}\left[d_{1} F(\omega r) \cos 2 \theta+d_{2} F(\omega r) \sin 2 \theta\right]
\end{aligned}
$$

where

$$
F(r)=\frac{1}{r} K_{1}(r)+\frac{1}{2} K_{0}(r)-\frac{1}{r^{2}} \quad \text { and } \quad \omega=\sqrt{\frac{2}{1-v}}
$$

with $K_{0}$ and $K_{1}$ as Bessel functions of the second kind of order zero and one, respectively. The associated stresses are written in a form to expose the singular nature of the doublet solution

$$
\begin{aligned}
& \Delta \sigma_{11}=\frac{E}{4 \pi}\left\{\begin{array}{l}
-d_{1}\left[\left(\frac{1}{r^{2}}+B_{1}(r)\right) \sin 2 \theta+\left(\frac{1}{r^{2}}+B_{2}(r)\right) \sin 4 \theta\right] \\
+d_{2}\left[\left(c_{1} \ln r+C_{1}(r)\right)+\left(\frac{1}{r^{2}}+B_{2}(r)\right) \cos 4 \theta\right]
\end{array}\right] \\
& \Delta \sigma_{22}=\frac{E}{4 \pi}\left\{\begin{array}{l}
-d_{1}\left[\left(\frac{1}{r^{2}}+B_{1}(r)\right) \sin 2 \theta-\left(\frac{1}{r^{2}}+B_{2}(r)\right) \sin 4 \theta\right] \\
+d_{2}\left[\left(c_{2} \ln r+C_{2}(r)\right)+2\left(\frac{1}{r^{2}}+B_{1}(r)\right) \cos 2 \theta-\left(\frac{1}{r^{2}}+B_{2}(r)\right) \cos 4 \theta\right]
\end{array}\right\} \\
& \Delta \sigma_{12}=\frac{E}{4 \pi}\left\{\begin{array}{l}
-d_{1}\left[\left(c_{3} \ln r+C_{3}(r)\right)-\left(\frac{1}{r^{2}}+B_{2}(r)\right) \cos 4 \theta\right] \\
+d_{2}\left[-\left(\frac{1}{r^{2}}+B_{1}(r)\right) \sin 2 \theta+\left(\frac{1}{r^{2}}+B_{2}(r)\right) \sin 4 \theta\right]
\end{array}\right\}
\end{aligned}
$$

The five functions $B_{i}(r)$ and $C_{i}(r)$ are regular functions of $r$ at $r=0$. These functions, along with the three constants $c_{i}$, are given in Appendix A. The $r^{-2}$ singularity in the stresses is seen to coincide with Eq. (16), and the next most singular terms are logarithmic. There are no contributions of order $r^{-1}$. All the other contributions are well behaved at the doublet origin. It should be emphasized that Eq. (19) holds for a dislocation pair on the $x_{1}$-axis. Results for other orientations of the pair can be obtained by coordinate transformation. 


\subsection{Formulation for a single straight crack}

Consider a crack in the film of length $2 a$ (i.e. of dimensional length $2 a l$ ) extending from $-a$ to $a$ along the $x_{1}$-axis. The prestress in the film is $\sigma_{\alpha \beta}^{0}=\sigma^{0} \delta_{\alpha \beta}$. A distribution of doublets, $d_{1}=0$ and $d_{2} \equiv d(\eta)$ for $|\eta|<a$, along $x_{1}$-axis canceling the normal traction due to the prestress can be used to construct the solution for the crack. The resulting integral equation for the $d(\eta)$ is

$$
\frac{E}{4 \pi} \int_{-a}^{a}\left[\frac{1}{(x-\eta)^{2}}+c_{2} \ln |x-\eta|+A(x-\eta)\right] d(\eta) \mathrm{d} \eta=-\sigma^{0} \quad \text { for } \quad|x|<a
$$

where $A(r)=C_{2}(r)+2 B_{1}(r)-B_{2}(r)$ is analytic at $r=0$. The representation (20) is formal in that the term containing the kernel, $(x-\eta)^{-2}$, is unbounded without special definition. A finite contribution can be defined to give the equation precise meaning (Kaya and Erdogan, 1987; Kaw, 1991; Willis and Nemat-Nasser, 1991). The solution to Eq. (20) will be given in the next section.

\subsection{An alternative formulation}

When the crack is curved, terms of order $r^{-1}$ arise in the kernel due to coordinate transformations along with those of order $r^{-2}$, and the existing numerical methods are not applicable as they stand. Therefore, it has been necessary to recast the equations by reducing the order of the singularity to a Cauchy-type singularity. Consider a crack of length $a$, straight or curved, and let $s$ be the length measured from one end with $\mathrm{d} s$ as the line element. Let $\mathbf{x}$ be any point in the plane off the crack, and let $\Delta \sigma_{\alpha \beta}(\mathbf{x})$ be the stress at that point due to the distribution of doublets $\mathbf{d}(s)$ along the crack. Write $\Delta \sigma_{\alpha \beta}(\mathbf{x})$ as

$$
\Delta \sigma_{\alpha \beta}(\mathbf{x})=\int_{0}^{a}\left[\Sigma_{\alpha \beta}^{0}\left(\mathbf{x}, \mathbf{x}^{\prime}\right) \cdot \mathbf{d}\left(s^{\prime}\right)+\Sigma_{\alpha \beta}^{1}\left(\mathbf{x}, \mathbf{x}^{\prime}\right) \cdot \mathbf{d}\left(s^{\prime}\right)\right] \mathrm{d} s^{\prime}
$$

where $\mathbf{x}^{\prime}$ is the position vector to the crack at $s^{\prime}$. Further, take $\sum_{\alpha \beta}^{0}$ to represent only the terms with singularity $r^{-2}$ in Eq. (19) (i.e. the stresses in Eq. (16)) and let $\sum_{\alpha \beta}^{1}$ represent the remaining terms. By virtue of the fact that Eq. (16) is the plane stress dislocation doublet solution, the contribution from the integrand, $\int \sum_{\alpha \beta}^{0} \cdot \mathbf{d} \mathrm{d} s$, is precisely the same as is obtained from distributing dislocations $\mathbf{b}(s)$ along the crack according to

$$
\int_{0}^{a}\left[S_{\alpha \beta}^{0}\left(\mathbf{x}, \mathbf{x}^{\prime}\right) \cdot \mathbf{d}\left(s^{\prime}\right)\right] \mathrm{d} s^{\prime}=\int_{0}^{a}\left[S_{\alpha \beta}^{0}\left(\mathbf{x}, \mathbf{x}^{\prime}\right) \cdot \mathbf{b}\left(s^{\prime}\right)\right] \mathrm{d} s^{\prime}
$$

where $\mathbf{b}(s)=-\mathrm{d} \mathbf{d} / \mathrm{d} s$ and $\mathbf{S}_{\alpha \beta}^{0}$ is the classical stress field of a dislocation in plane stress.

With $\mathbf{d}(s)=\int_{s}^{a} \mathbf{b}\left(s^{\prime \prime}\right) \mathrm{d} s^{\prime \prime}$, the second contribution in Eq. (21) is 


$$
\int_{0}^{a}\left[\Sigma_{\alpha \beta}^{1}\left(\mathbf{x}, \mathbf{x}^{\prime}\right) \cdot \mathbf{d}\left(s^{\prime}\right)\right] \mathrm{d} s^{\prime}=\int_{0}^{a}\left[\Sigma_{\alpha \beta}^{1}\left(\mathbf{x}, \mathbf{x}^{\prime}\right) \cdot \int_{s}^{a} \mathbf{b}\left(s^{\prime \prime}\right) \mathrm{d} s^{\prime \prime}\right] \mathrm{d} s^{\prime}
$$

Noting that the integration on the right-hand side of Eq. (23) is over a triangle in the $\left(s^{\prime}, s^{\prime \prime}\right)$ plane, interchange the order of integration to obtain

$$
\int_{0}^{a}\left[\Sigma_{\alpha \beta}^{1}\left(\mathbf{x}, \mathbf{x}^{\prime}\right) \cdot \mathbf{d}\left(s^{\prime}\right)\right] \mathrm{d} s^{\prime}=\int_{0}^{a}\left[\boldsymbol{S}_{\alpha \beta}^{1}\left(\mathbf{x}, \mathbf{x}^{\prime}\right) \cdot \int_{s}^{a} \mathbf{b}\left(s^{\prime \prime}\right) \mathrm{d} s^{\prime \prime}\right] \mathrm{d} s^{\prime}
$$

where

$$
\mathbf{S}_{\alpha \beta}^{1}\left(\mathbf{x}, \mathbf{x}^{\prime \prime}\right)=\int_{0}^{s\}} \Sigma_{\alpha \beta}^{1}\left(\mathbf{x}, \mathbf{x}^{\prime}\right) \mathrm{d} s^{\prime}
$$

It follows, therefore, the doublet representation (21) can be replaced by an integral of a distribution of dislocations $\mathbf{b}(s)$ according to

$$
\sigma_{\alpha \beta}(\mathbf{x})=\int_{0}^{a}\left[\mathbf{S}_{\alpha \beta}^{0}\left(\mathbf{x}, \mathbf{x}^{\prime}\right)+\mathbf{S}_{\alpha \beta}^{1}\left(\mathbf{x}, \mathbf{x}^{\prime}\right)\right] \cdot \mathbf{b}\left(s^{\prime}\right) \mathrm{d} s^{\prime}
$$

where $\mathbf{S}_{\alpha \beta}^{0}$ is the stress distribution (23) for a dislocation with singularity ( $\mathbf{x}-$ $\left.\mathbf{x}^{\prime}\right)^{-1}$ and the contribution to the kernel from $\mathbf{S}_{\alpha \beta}^{1}\left(\mathbf{x}, \mathbf{x}^{\prime}\right)$ is bounded as $\mathbf{x} \rightarrow \mathbf{x}^{\prime}$. Integral equations formed from Eq. (26) are of the classical Cauchy-type and directly amenable to numerical solution by methods such as those detailed by Erdogan and Gupta (1972). The relation of the stress intensity factors to the dislocation distributions at the ends of the cracks is the same as in the classical plane stress formulation.

\section{Straight cracks}

Solutions to a number of problems for straight film cracks will be presented in this section. With two exceptions, where analytical results have been obtained, the solutions have been obtained numerically based on one of the two integral equation formulations described in the previous section. The selection of problems is intended to provide insight into interactions between film cracks and their role in establishing cracking patterns. The subsections consider: a single finite crack; arrays of parallel semi-infinite cracks; mixed mode interactions among parallel cracks; and interactions between perpendicular cracks.

\subsection{Single finite crack}

The integral equation (20) employing the doublet distribution was solved numerically using methods developed in the references cited earlier. The prestress in the film is equi-biaxial tension with $\sigma_{\alpha \beta}^{0}=\sigma^{0} \delta_{\alpha \beta}$. Symmetry dictates the crack to be in mode I $\left(K_{\mathrm{II}}=0\right)$. The near tip field is thus characterized by $K_{\mathrm{I}}$. Results will 
also be presented for the contribution from the next order in the hierarchy of crack tip fields, the $T$-stress, $\sigma_{11}=T$, which is finite at the tip. The dependence of $K_{\mathrm{I}} / \sigma^{0} \sqrt{l}$ and $T / \sigma^{0}$ on the normalized crack half-length, $a / l$, is shown in Fig. 2. The results have been computed for $v=0.3$, as will be the case for all the numerical results presented in the paper. For $a / l \cong 1$, the stress intensity factor is already close to the asymptotic limit for a semi-infinite crack

$$
\frac{K_{\mathrm{I}}}{\sigma^{0} \sqrt{l}}=\sqrt{\left(1-v^{2}\right)}
$$

which is obtained from Eqs. (10)-(12). Recall that $l$ has been defined in Eq. (12) such that this limiting result is exact. The trend shown in Fig. 2 is in accord with the three-dimensional result of Nakamura and Kamath (1992) quoted in Section 1. One end of the crack ceases to be affected by the other end when the crack length is larger than about $2 l$, i.e., about four times the film thickness in the absence of elastic match between the film and substrate. The elastic mismatch between the film and the substrate makes its presence felt through $l$, which is plotted as $l / h$ in Fig. 1.

The $T$-stress is also plotted in Fig. 2. The prestress component $\sigma_{11}^{0}=\sigma^{0}$ acting parallel to the crack is accounted for in this result. (When the prestress is not equi-biaxial, the effect of $\sigma_{11}^{0}$ on $T$ is readily adjusted by superposition. This same component has no effect on $K_{\mathrm{I}}$.) The interaction of the crack with the substrate gives rise to a positive $T$-stress. The corresponding plane stress problem for a sheet unattached to a substrate tension has $T=0$. A positive $T$-stress has implications for the configurational stability of the straight crack (Cotterell and Rice, 1980), giving rise to the possibility that small perturbations may cause the crack path to depart from its initial direction. A stability analysis for the full film/

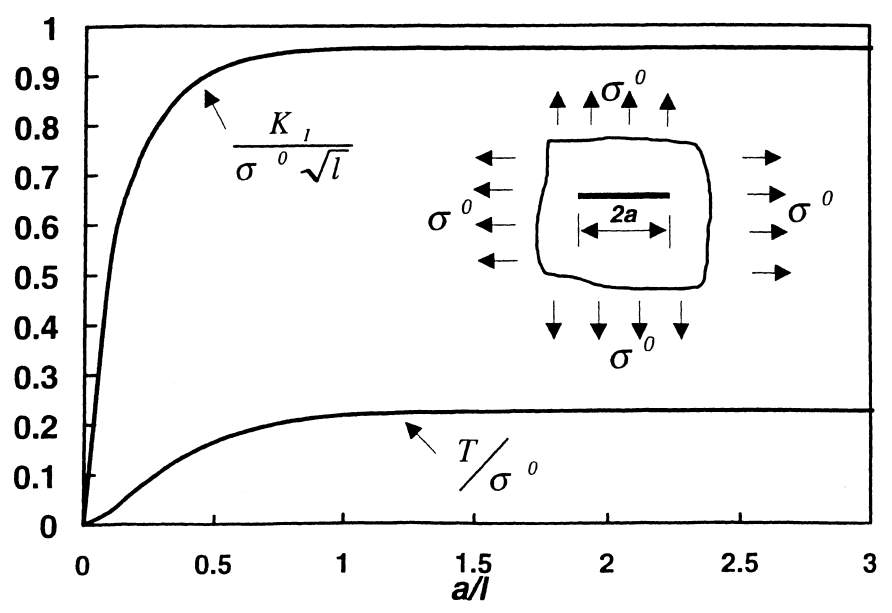

Fig. 2. Stress intensity factor and $T$-stress for a film crack subject to equi-biaxial tension $(v=0.3)$. 
substrate model has not been performed, and thus the role of $T$ may not be the same as for a free standing film.

\subsection{Parallel semi-infinite cracks, including sequential cracking}

Consider the infinite array of equally spaced semi-infinite film cracks with aligned tips shown in Fig. 3. Symmetry again dictates that each crack tip is in mode I. The energy release rate of each crack tip is readily calculated by imagining all the cracks to advance by an unit increment, thereby, equivalently, transferring a unit increment of the film far ahead of the tips to the remote wake. The solution to Eq. (13) in the remote wake depends only on $x_{2}$ and is easily produced. The requisite energy accounting gives

$$
G=\frac{l \sigma^{0^{2}}}{\bar{E}} \tanh \left(\frac{H}{2 l}\right) \quad \text { or } \quad \frac{K_{\mathrm{I}}}{\sigma^{0} \sqrt{l}}=\sqrt{\left(1-v^{2}\right)} \sqrt{\tanh \left(\frac{H}{2 l}\right)}
$$

This result approaches the result for an isolated semi-infinite crack in Eq. (10) or Eq. (27) when the crack spacing $H$ exceeds approximately $3 l$. Elastic mismatch, appearing through $l$, has a fairly strong influence on the interaction. Cracks in a film which is stiff relative to its substrate interact across greater distances than vise versa.

Exact results for $G$ for the array of equally spaced cracks channeling in steadystate across the film can be obtained from the plane strain solution for periodic

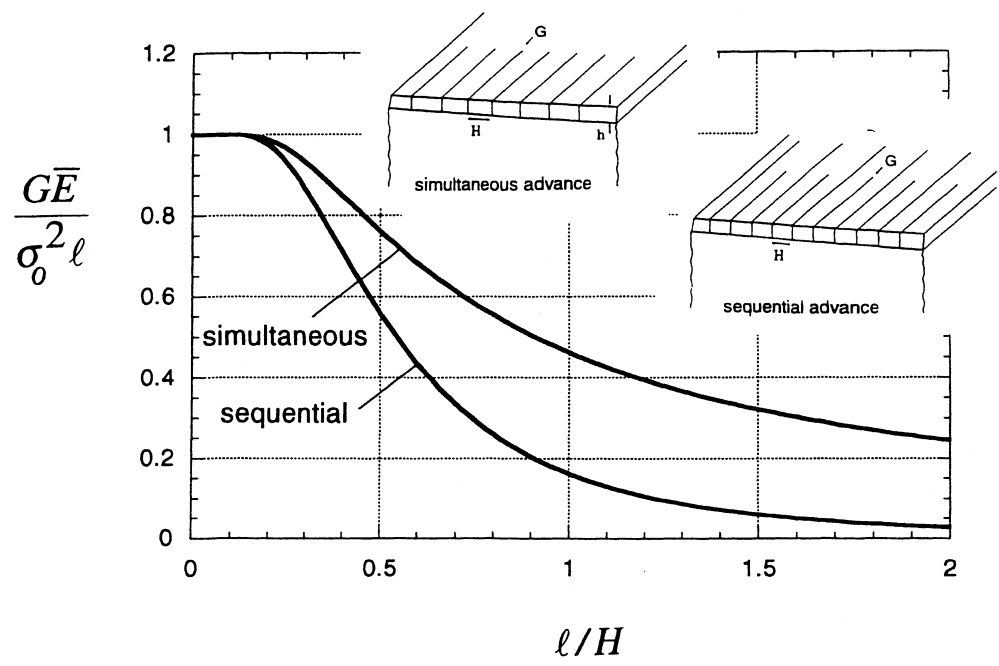

Fig. 3. Energy release rate at each crack tip for steady-state channeling of parallel film cracks. The upper curve applies to simultaneous advance of all the cracks, while the lower curve applies to the sequential process where a new set of cracks propagates midway between a previously formed set of cracks. 
edge cracks in the film. Denote the solution for the energy release rate for the two-dimensional plane strain problem for cracks of length $a$ (where $a \leq h$ ) with spacing $H$ by $G_{p s}(a)$. A simple energy argument (Hutchinson and Suo, 1992) provides the connection between the two energy release rates as

$$
G=\frac{1}{h} \int_{0}^{h} G_{p s}(a) \mathrm{d} a
$$

Solutions for $G_{p s}(a)$ are not available for periodic crack arrays in films with elastic properties which differ from the substrate, but accurate numerical results are available for homogeneous, isotropic elastic solids (Tada et al., 1985). Evaluation of Eq. (29) using these accurate numerical results reveals that the approximate estimate of $G$ for the present model (28) for the case of no elastic mismatch is accurate to within a few percent over the entire range of $H / l$.

The energy release rate for an idealized sequential cracking process which is more in accord with the way film cracks appear can also be obtained simply. Consider the semi-infinite mode-I cracks in Fig. 3 advancing midway between previously formed infinite cracks. Because the solution to Eq. (13) is far ahead and far behind the current location of the crack tips is elementary, it is very simple to do the energy accounting necessary to obtain the energy released by each semi-infinte crack. The results is

$$
G=\frac{l \sigma^{0^{2}}}{\bar{E}}\left[2 \tanh \left(\frac{H}{2 l}\right)-\tanh \left(\frac{H}{l}\right)\right]
$$

Alternatively, following the procedure of Hutchinson and Suo (1992), denote the result from Eq. (28) for aligned semi-infinite cracks in Fig. 3 spaced a distance $2 H$ apart by $G^{0}(2 H)$. The energy released by propagation of the first set of cracks far ahead of the current set of crack tips in Fig. 3 is $G^{0}(2 H)$, per height $2 H$ in the $x_{2}$ direction, while that released far behind the tips is $2 G^{0}(H)$ per height $2 H$. The energy release rate per tip is therefore precisely $G=2 G^{0}(H)-G^{0}(2 H)$. Eq. (30) follows immediately using Eq. (28). It is worth recording that the connection $G=$ $2 G^{0}(H)-G^{0}(2 H)$ is exact within the context of three-dimensional elasticity. Any error in Eq. (30) follows from the fact that Eq. (28) is derived from the present model which approximates the film/substrate interaction for the periodic cracks.

The two results, (28) and (30), are compared in Fig. 3. The energy release for cracks nucleated sequentially is significantly less than the prediction for an array of crack imagined to appear simultaneously. This difference has important implications for the relation between crack spacing and residual stress. Delannay and Warren (1991) and Thouless et al., 1992 carried out experiments to obtain the evolution of average crack spacing in brittle films under conditions where the prestress was continuously increased. In both papers, the experiential data was compared to a theoretical prediction for sequential cracking similar, but not identical to Eq. (30). If $\Gamma_{\mathrm{c}}$ is the mode-I film toughness and if $2 H$ is the current crack spacing, then Eq. (30) with $G=\Gamma_{\mathrm{c}}$ specifies the stress $\sigma^{0}$ at which the next 
set of cracks will channel halfway between the current set according to the idealized sequential processes.

\subsection{Mixed mode interactions among parallel cracks}

The problems posed in this subsection are intended to provide insight into cracking trajectories when film cracks are within interaction distance. The primary emphasis is the mix of mode I and II at the tip, from which the direction of crack advance can be inferred. The fracture properties of the film are taken to be isotropic. The solutions to the problems posed below were produced using the method of Section 3.3. As in the previous two subsections, it is assumed that the prestress in the film is equi-biaxial tension. However, the component $\sigma_{11}^{0}$ has no influence on the stress intensity factors in these problems if the cracks are parallel to the $x_{1}$.

A pair of aligned film cracks of length $2 a$ lying side by side a distance $2 H$ apart is considered in Fig. 4. Stress intensity factors, $K_{\mathrm{I}}$ and $K_{\mathrm{II}}$, of the right-hand tip of the upper crack are shown. There is some interaction when $H / l=2$, but, for $H / l=5, K_{\mathrm{I}}$ is nearly identical to the result for the isolated crack in Fig. 2 and $K_{\mathrm{II}} \cong 0$. The cracks become effectively semi-infinite as far as the stress intensity factors are concerned when $a / l>2$. The mode-II stress intensity factor $K_{\text {II }}$ of the upper, right-hand tip is negative. This implies that a putative crack advancing from that tip would kink upward. Crack paths evolving from these two starter cracks will spread apart rather than come together. In this sense, the two aligned cracks 'repel' each other.

Results illustrating the behavior expected for cracks propagating towards each other can be inferred from the results displayed in Fig. 5. A semi-inifinite crack advancing to the right encounters two aligned semi-infinite cracks advancing in the opposite direction. The middle crack lies precisely half way between the two outer cracks and is therefore in mode I. The outer cracks shield the middle crack (Fig. 5(a)) when the tip of the middle crack passes between the outer cracks. The stress intensity factors for the upper crack are shown in Fig. 5(b) and (c). The energy release rate (not shown) of this crack also drops as the tips pass each other. The mode-II stress intensity factor of the upper crack changes sign as the tips pass each other. (For the upper crack with the tip pointing to the left, a positive $K_{\mathrm{II}}$ is defined such that it produces a positive shear stress component, $\sigma_{12}$, directly to the left of the tip.) The trend of $K_{\text {II }}$ shown in Fig. 5(c) implies that the outer cracks tend to veer away from the middle crack as the tips approach each other, and then switch direction and veer toward the middle crack after the tips have passed.

Crack paths in unsupported thin sheets and plates have been addressed using elastic fracture mechanics by a number of authors. In particular, Melin (1983) has shown that the behavior noted above for film cracks approaching each other also occurs in brittle free standing sheets. Broberg (1987) provides general discussion of crack paths and a comprehensive list of references. 


\subsection{Interaction between perpendicular cracks}

Consider a semi-infinite crack approaching an infinite crack at a right angle as shown in Fig. 6(a). The pre-stress in the film is equi-biaxial tension $\sigma^{0}$. Symmetry dictates the advancing crack to be mode I. When the tip of the advancing crack is within $3 l$ from the other crack, its stress intensity factor begins to drop below Eq. (27), falling to a minimum at a distance of $0.3 l$. The intensity factor then increases sharply as the remaining ligament is reduced to zero. The present model may not be reliable in the range for $x / l<0.3$. Three-dimensional effects are expected to become important when size of the controlling region is of the order of the film thickness. The trend in Fig. 6(a) suggests that an advancing crack may arrest at a

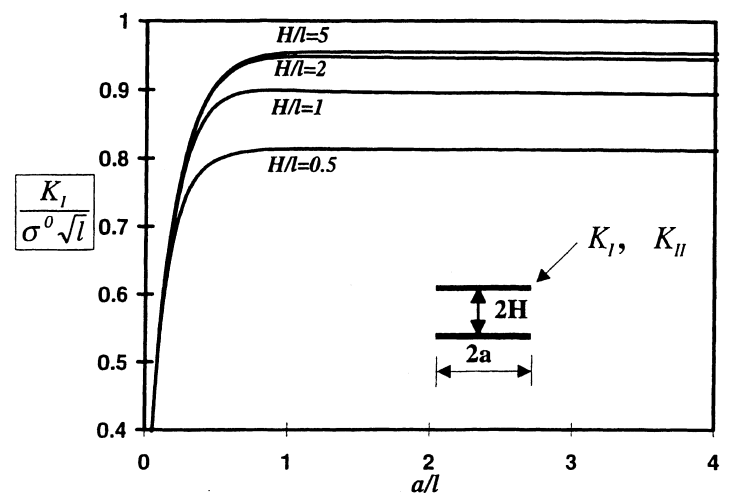

(a)

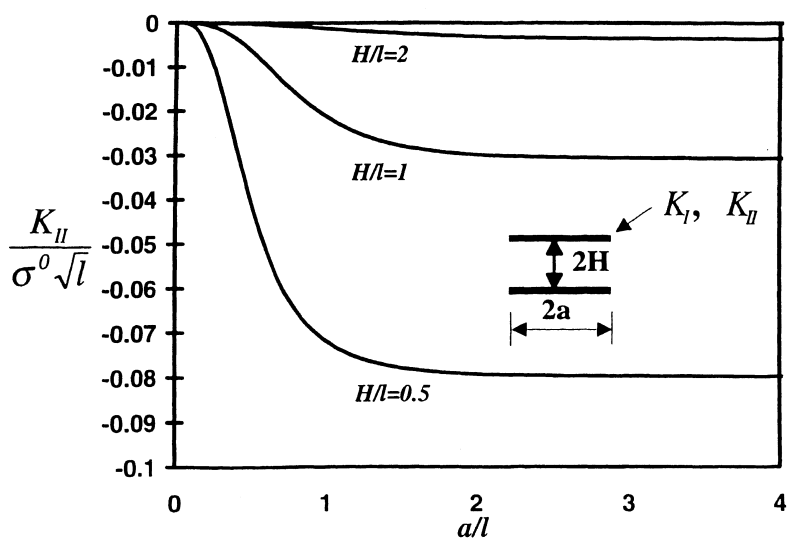

(b)

Fig. 4. Mode I and II stress intensity factors for the upper right hand crack tip for two aligned parallel cracks $(v=0.3)$. 

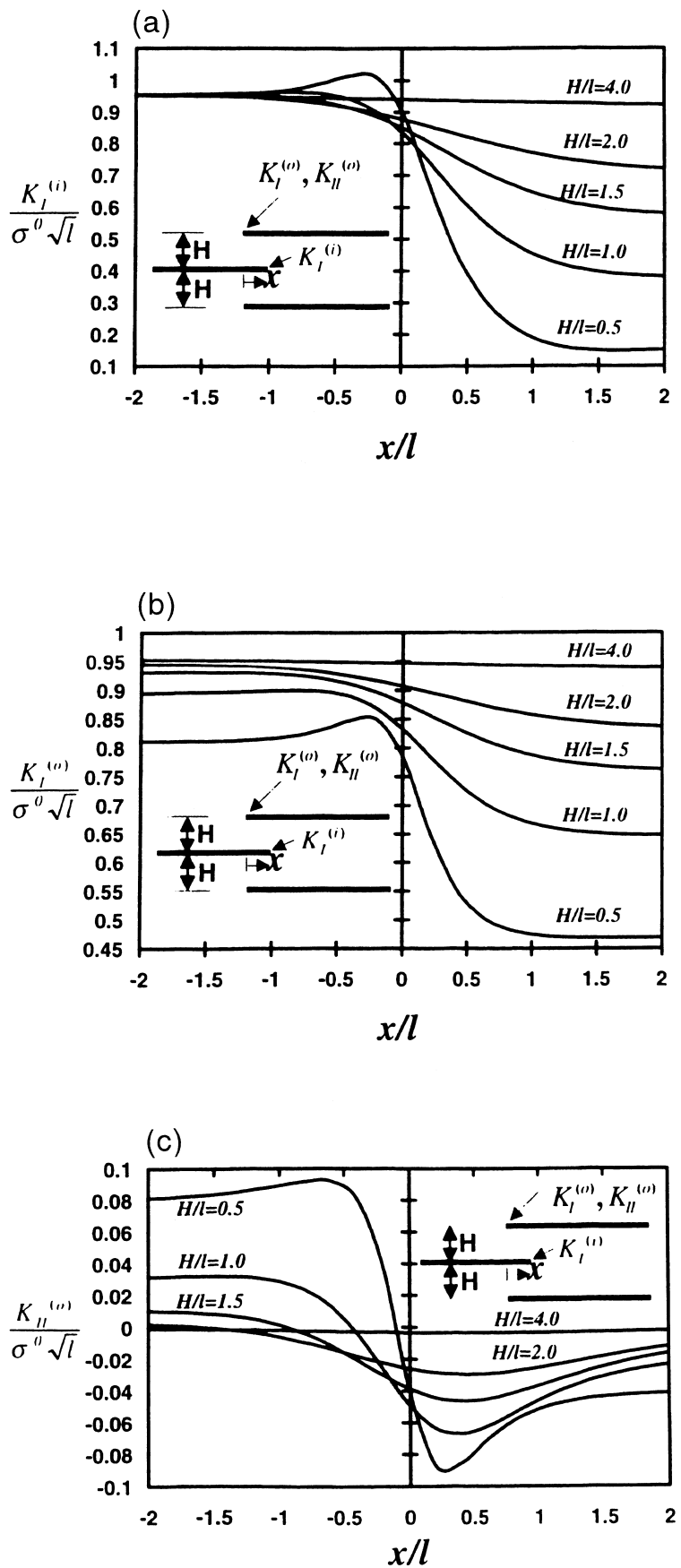

Fig. 5. Stress intensity factors for parallel cracks approaching one another $(v=0.3)$. 
distance on the order of $l / 2$ from the crack it is approaching due to the drop in stress intensity below the steady-state value.

The companion result for a film crack emerging at a right angle from an infinite crack (or a free edge) is given in Fig. 6(b). In this case, the stress intensity factor increases monotonically nearly attaining steady-state (28) when its tip has advanced by about $l$.

\section{Curved cracks}

In this section, the possibility of the existence of curved crack trajectories will

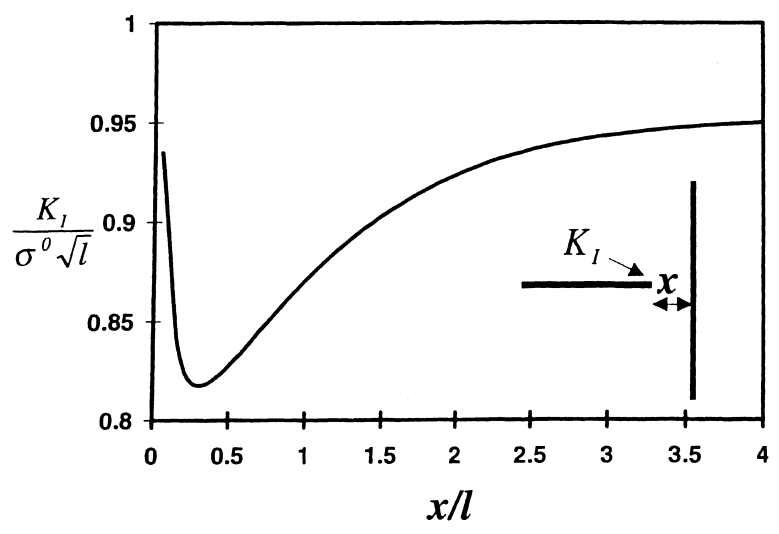

(a)

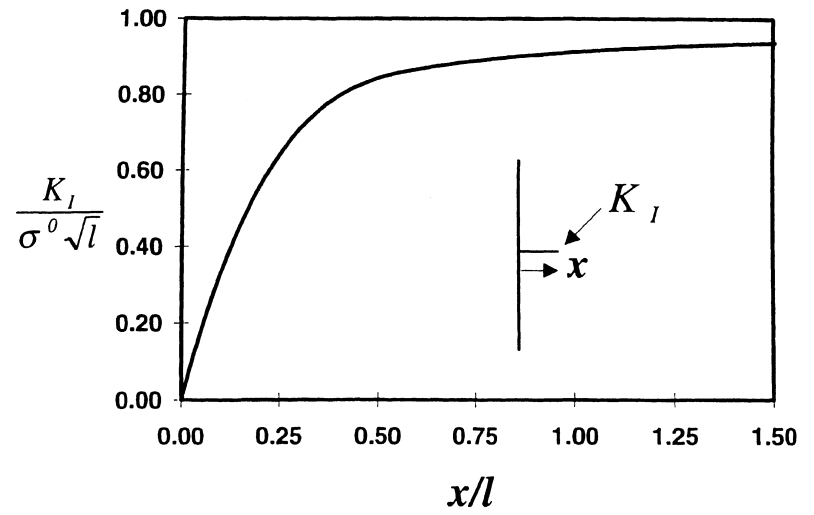

(b)

Fig. 6. Stress intensity factor: (a) a crack tip approaching a perpendicularly aligned crack, and (b) a crack tip emerging at right angles from a long crack $(v=0.3)$. 
be explored, and it will be demonstrated that a crack propagating in a spiral path is possible.

\subsection{Circular arc cracks}

Consider the film crack in Fig. 7 in the shape of a circular arc of radius $R$ with subtended angle $2 \theta$. The prestress in the film is equi-biaxial tension $\sigma^{0}$. The integral equation formulation of Section 3.3 was used to generate the numerical results for the stress intensity factors which are presented for the right hand tip. For cracks having $R / l \geq 5$, the curvature ceases to have any noticeable effect, and near mode I conditions prevail at the tip. At crack radii less than this level, the energy release rate falls below the rate for an isolated straight crack (27). The sign of $K_{\mathrm{II}}$ is such that the crack would kink or turn outward from the circular arc, i.e.

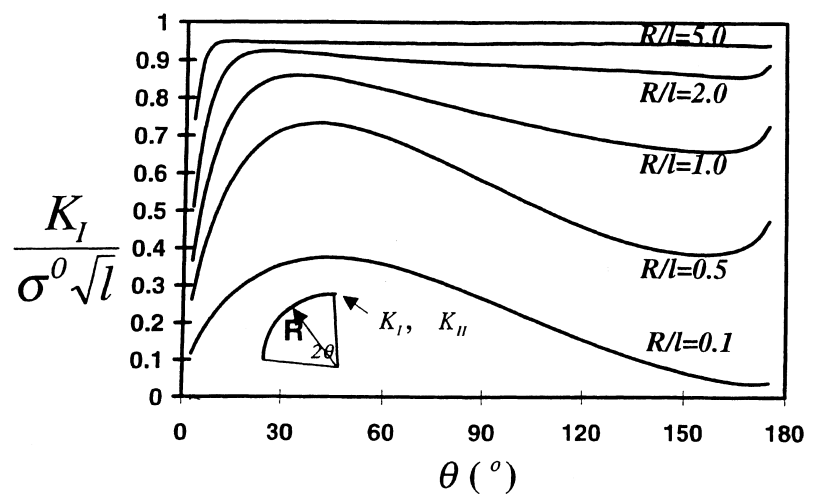

(a)

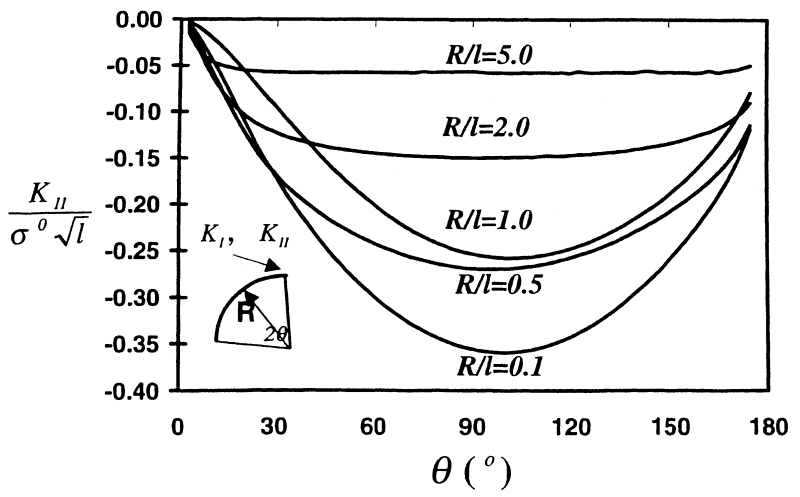

(b)

Fig. 7. Stress intensity factors for a circular film crack $(v=0.3)$. 
upwards in Fig. 7. This is the same trend predicted for a plane stress crack in a sheet under equi-biaxial biaxial stressing. The plane stress results for a circular arc in a biaxially stressed thin sheet (Tada et al., 1985) are approached by the present solution when $R / l$ becomes very small, providing a check on the present numerical procedures. The curves in Fig. 7 for $R / l=0.1$ are reasonably well approximated by this limiting result. This small cracks are not expected to realistically characterized by the present model because three-dimensional effects become dominant.

The sign of $K_{\text {II }}$ of the circular arc crack is such it would evolve in such a way that it would reduce its curvature and asymptote to a straight path once critical conditions are met. When this observation is combined with the tendency of a tip of a straight crack to be 'attracted' to any crack it parallels (cf. Fig. 5), a clue for starting conditions which might lead to a spiraling crack emerges. Consider the two circular arc cracks having common center shown in Fig. 8. The inner crack is sufficiently long (subtending a total angle of $180^{\circ}$ ) such that its ends do not interact with each other or with the ends of the outer crack. It could equally well be taken as a full circular crack. The radius of the outer crack is $R+H$, and its length is also sufficiently great such that the intensity factors at one end have no effect on those at the other end. When $H$ is small enough, the tip of the outer crack is expected to be attracted to the inner crack, on the grounds cited above. However, as $H$ increases, the sign of $K_{\mathrm{II}}$ must change such that when $H$ is large enough the tip will deflect outward when propagation occurs. By continuity, there must exist a spacing $H$ such that $K_{\mathrm{II}}=0$. At this spacing, the tip of the outer crack will advance smoothly in a circular arc and maintain its distance $h$ from the inner crack, either until it senses the ends of the inner crack or it senses its own ends had the inner crack been taken as a full circle.

The problem posed for the circular arc cracks in Fig. 8 has been solved using the method of Section 3.3. For specified values of $R / l$, the stress intensity factors for the tips of the outer crack were computed as a function of $H / l$. The value of $H / l$ for which $K_{\mathrm{II}}=0$ was determined. This value is plotted as a function of $R / l$ in Fig. 8(a) over the range of values for which the model is expected to have physical validity. The corresponding value of $K_{\mathrm{I}}$ is shown in Fig. 8(b). No corresponding location exists with $K_{\mathrm{II}}=0$ when the shorter crack lies inside the longer crack. Then, the two trends cited in the previous paragraph each act so as to attract the tip of the shorter crack towards the longer crack.

The results of Fig. 8(a) for the location of a mode-I tip are now employed to make an approximate prediction for the path of a spiral crack. The fundamental assumption is that the crack tip must maintain mode-I conditions at its tip ( $K_{\mathrm{II}}=$ $0)$ as it advances. Denote the result for spacing between the tip and the inner crack in Fig. 8 (a) by $H / l=f(R / l)$. In planar polar coordinates $(r, \theta)$, let $r(\theta)$ be the equation of the spiral where $\theta$ increases monotonically. The path is approximated by the following equation:

$$
\frac{r(\theta)}{l}-\frac{r(\theta-2 \pi)}{l}=f\left[\frac{r(\theta-2 \pi)}{l}\right]
$$


where the tip is currently at $(r(\theta), \theta)$. In applying the solution of Fig. 8(a), the distance $H$ has been identified as $r(\theta)-r(\theta-2 \pi)$, and the radius of curvature of the loop of the crack path is directly opposite the tip is approximated as $r(\theta-2 \pi)$. An initial spiral-like loop must pre-exist to initiate a full spiral. As an illustration, assume an initial spiral crack has the form

$$
\frac{r}{l}=\frac{r_{0}}{l}+f\left[\frac{r_{0}}{l}\right] \frac{\theta}{2 \pi} \quad \text { for } \quad 0 \leq \theta \leq 2 \pi
$$

The starter loop (32) only satisfies (31) exactly at $\theta=2 \pi$. For $\theta \geq 2 \pi$, Eq. (32) is used to generate the spiral. An example is shown in Fig. 9 for the starting condition $r_{0} / l=3$. It is evident that the approximation (31) will be virtually

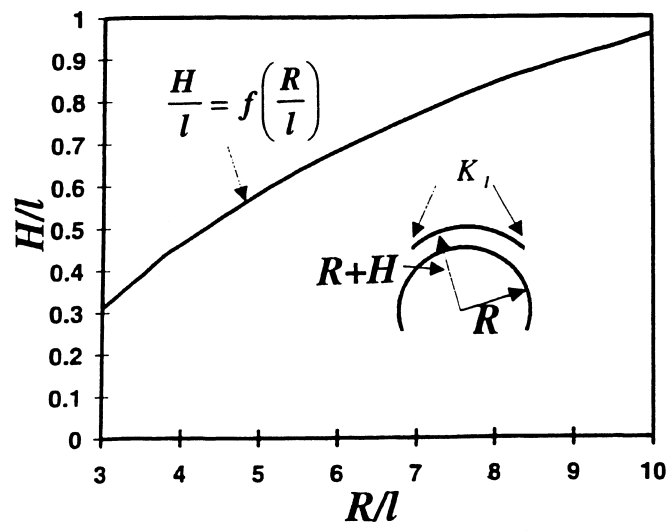

(a)

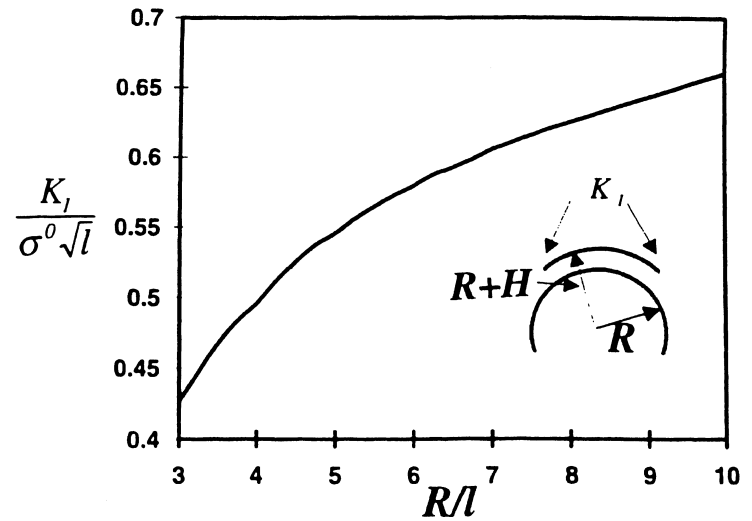

(b)

Fig. 8. Standoff $H$ for $K_{\mathrm{II}}=0$ and associated mode-I stress intensity factor for a circular arc crack outside a circular crack $(v=0.3)$. 
unaffected if the actual radius of curvature of the loop segment opposite the tip is used in place of $r(\theta-2 \pi)$.

Arguments for the existence of spiral cracks have been made on the grounds that they supply a very efficient mechanism for relieving the elastic energy stored in the film. That argument is insufficient, however, because it does not take into account the fact that a crack in a brittle film grows at its tip, advancing continuously such that mode-I conditions are maintained. The present model shows that spiral mode-I paths can exist in biaxially stresses films if spiral-shaped flaws are present to get them started. An isolated spiral crack was photographed by Argon (1959) in a surface layer under residual tension on a Pyrex glass plate. We are indebted to Argon for calling this pattern to our attention as it provided the initial motivation behind the present effort to seek a theoretical explanation for spirals. Profuse spiral tunnel cracking has been observed by Dillard et al. (1994) in brittle adhesive layers bonding together glass plates. An example is displayed in Fig. 10, where the crack is photographed through one of the plates. As the adhesive cures, biaxial tensile stress develops first producing 'mud cracks' that subdivide the adhesive into polygonal regions. With further curing, spiral cracks nucleate and form within the polygonal regions. Most of these cracks appeared to nucleate at or near the polygonal boundary and then spiral inward. These cracks are unlike the film cracks contemplated in the present model in other respects as well. While the 'mud cracks' extend all the way through the adhesive at right angles to the adhesive/glass interfaces, each spiral crack is confined near one interface and propagates at an inclined angle to the interface. It is this inclination which makes the crack opening appear so large.

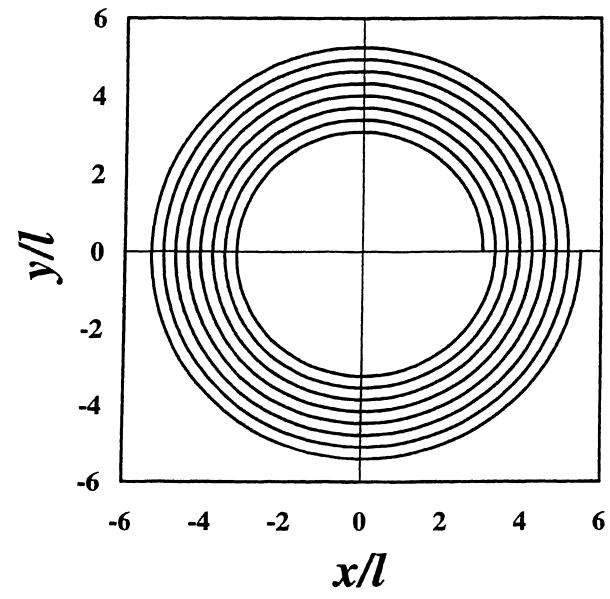

Fig. 9. Spiral crack pattern satisfying $K_{\mathrm{II}}=0$ as it evolves $(v=0.3)$. 


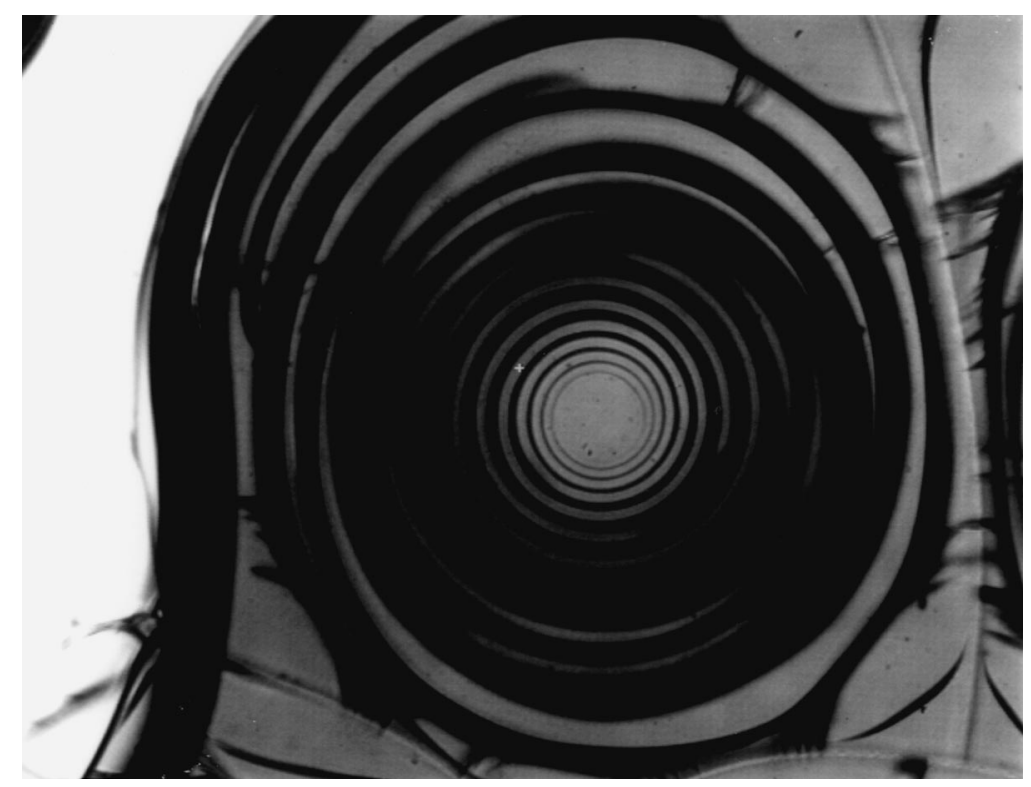

Fig. 10. Spiral crack in brittle adhesive bonding two glass plates (Dillard et al., 1994).

\section{Acknowledgements}

This work was supported in part by NSF Grants CMS-96-34632 and DMR-9400396 and in part by the Division of Engineering and Applied Sciences, Harvard University.

\section{Appendix A. Details of the doublet solution}

The functions $B_{i}(r)$ and $C_{i}(r)$ in Section 3.1 are defined as follows.

$$
\begin{aligned}
& C_{1}(r)=\left[\frac{1-v}{4(1+v)}-\frac{1+v}{2(1-v)}\right] K_{0}(r)+\frac{1}{1-v^{2}} K_{0}(\omega r)-c_{1} \ln r \\
& C_{2}(r)=-\left[\frac{1-v}{4(1+v)}+\frac{1+v}{2(1-v)}\right] K_{0}(r)-\frac{1}{1-v^{2}} K_{0}(\omega r)-c_{2} \ln r \\
& C_{3}(r)=\frac{1}{2}\left[C_{1}(r)-c_{2}(r)\right] \\
& B_{1}(r)=\left[\frac{K_{1}(r)}{r}+\frac{1}{2} K_{0}(r)\right]-\frac{1}{r^{2}}
\end{aligned}
$$




$$
B_{2}(r)=\frac{2}{1+v}\left[(1-v) \frac{A_{1}(r)}{r^{2}}-2 \frac{A_{1}(\omega r)}{r^{2}}\right]-\frac{1}{r^{2}}
$$

where

$$
A_{1}(r)=\frac{1}{8}\left[r^{2} K_{0}(r)+8 r K_{1}(r)+48 F(r)\right]
$$

and

$$
c_{1}=\frac{v^{2}+6 v-3}{4\left(1-v^{2}\right)}, \quad c_{2}=\frac{3 v^{2}+2 v+7}{4\left(1-v^{2}\right)}, \quad c_{3}=\frac{1}{2}\left(c_{1}-c_{2}\right)
$$

\section{References}

Argon, A.S., 1959. Surface cracks on glass. Proc. Roy. Soc. A250, 472-481.

Beuth, J.L., 1992. Cracking of thin bonded films in residual tension. Int. J. Solids Structures 29, 16571675.

Beuth, J.L., Klingbeil, N.W., 1996. Cracking of thin films bonded to elastic-plastic substrates. J. Mech. Phys. Solids 44, 1411-1428.

Broberg, K.B., 1987. On crack paths. Engng. Fracture Mech. 28, 663-679.

Chen, S.-Y., Chen, I.-W., 1995. Cracking during pyrolysis of oxide thin films-phenomenology, mechanisms and mechanics. J. Am. Ceram. Soc. 78, 2929-2939.

Cotterell, B., Rice, J.R., 1980. Slightly curved or kinked cracks. Int. J. Fracture 16, 155-169.

Delannay, F., Warren, P., 1991. On crack interaction and crack density in strain-induced cracking of brittle films on ductile substrates. Acta Metall. Mater. 39, 1061-1072.

Dillard, D.A., Hinkley, J.A., Johnson, W.S., St.Clair, T.L., 1994. Spiral tunneling cracks induced by environmental stress cracking in LARC-TPI adhesives. J. Adhesion 44, 51-67.

Erdogan, F., Gupta, G.D., 1972. On the numerical solution of singular integral equations. Q. Appl. Math., 523-534.

Garino, T.J., 1990. The cracking of sol-gel films during drying. In: Mat. Res. Soc. Symp. Proc., Materials Research Society, vol. 180, 497-502.

Hu, M.S., Evans, A.G., 1989. The cracking and decohesion of thin films on ductile substrates. Acta Metall. 37, 917-925.

Hutchinson, J.W., Suo, Z., 1992. Mixed mode cracking in layered materials. Adv. Appl. Mech. 29, 63191.

Kaya, A.C., Erdogan, F., 1987. On the solution of integral equations with strongly singular kernels. Q. Appl. Math. 45, 105-122.

Kaw, A.K., 1991. On evaluating integrals with strongly singular integrands. Adv. Eng. Software 13, 84-101.

Melin, S., 1983. Why do cracks avoid each other? Int. J. Fracture 23, 37-45.

Nakamura, T., Kamath, S., 1992. Three-dimensional effects in thin film fracture. Mech. Materials 13, 67-77.

Rice, J.R., 1968. Mathematical analysis in the mechanics of fracture. In: Leibowitz, H. (Ed.), Fracture, vol. 2. Academic Press, New York, pp. 191-311.

Tada, H., Paris, P.C., Irwin, G.R., 1985. The Stress Analysis of Cracks Handbook. Del Research Corp, St Louis, MO.

Thouless, M.D., Olsson, E., Gupta, A., 1992. Cracking of brittle films on elastic substrates. Acta Metall. Mater. 40, 1287-1292. 
Ye, T., Suo, Z., Evans, A.G., 1992. Thin film cracking and the role of substrate and interface. Int. J. Solids Structures 29, 2639-2648.

Willis, J.R., Nemat-Nasser, S., 1991. Singular perturbation solution of a class of singular integral equations. Q. Appl. Math. 48, 741-753. 